Case report

\title{
Achondroplastic Syndrome in a West African Dwarf Lamb
}

\author{
*AJADI R.A; AJALA 0.0; OYEYEMI M.O; AKUSU M.O \\ Department of Veterinary Surgery \& Reproduction, University of Ibadan. Ibadan, Nigeria
}

\author{
Received: April, 2004 \\ Accepted: November, 2004
}

\begin{abstract}
A three week old, male, West African Dwarf (WAD) lamb presented with disproportionate hind limb was diagnosed of having achondroplastic syndrome by physical and radiological examination. Physical examination showed the right hind limb was deformed at the level of the tarsus, metatarsus and phalanges. Radiological examination showed that the tarsal bones were small and laterally compressed, while the lateral sesamoid bone was absent. The right metatarsal bones were rudimentary while the phalanges were only represented by soft tissue structure. The serum alkaline phosphatase (ALP), aspartate transaminase (AST) and alanine transarninase (ALT) were higher in the achondroplastic lamb than the normal flock mate used as control. Based on the radiographic findings, it was concluded that ectrodactyly was the only feature of the achondroplastic syndrome in the lamb.
\end{abstract}

Key words:

Achondroplastic Syndrome, Lamb, Dwarf

\section{INTRODUCTION}

Congenital defects are abnormalities of structure or function present at birth (Leipold et al, 1972). They can affect an isolated portion of the body system, the complete system or several systems. The frequency of congenital defect in sheep is difficult to assess (Saperstein et at, 1975). The defects are peculiar to a breed, geographical location or environmental condition (Dennis, 1974; 1975).

Developmental errors, which ultimately are expressed in the skeleton, may be primary abnormalities of bones, cartilage or primitive mesenchymal cells (Palmer, 1993). They may be genetic or conditioned by the environment, and in ether case, the anomalies may be local or systemic. Genetic factors are characterized either by chromosomal aberrations or mutant genes (Rook et al, 1988). Environmental factors include viral agents, bacterial agents, drugs and plant teratogenes.

Heredita ry chondrodysplasia have been shown to be due to simple recessive inheritance (Rook et al, 1986; 1988). Two clinical entities have been reported to include those that were grossly abnormal at birth and those that developed abnormal conformation 4-6 weeks of age (Rook. et al, 1988). Radiographically, however, both groups of lambs have similar skeletal defects at births, substantiating a congenital defect.

Wray et al (1971) had described an achondroplastic syndrome in which ectrodactyly was a feature in South Country Cheviout Lambs. The principal features were achondroplasia of the head with protruding eyes, short ears and tail, with the hind hooves reduced in size and abnormal in shape. From available literature, there are no reports of congenital chondrodysplasia in the West African Dwarf Lamb. This report presents a case of an achondroplastic syndrome in a three week old lamb in which ectrodactyly is the main feature.

\section{Case History and Clinicopathological Findings:}

A three week old male West African Dwarf Lamb was presented to the Veterinary teaching hospital with complaint of disproportionate hind limbs. Careful questioning and review of the record of the sock revealed that the lamb belonged to a ewe in a flock of 28 sheep comprising 24 ewes and 4 rams. . The flocks are raised semi-intensively and breeding is uncontrolled and may occur during pasture grazing or in the pen. The ewe had lambed twice previously with no record of abortion or congenital defect in any of the previous lambs, similarly, there are no previous records of 
abortions or congenital defect in the entire flock. The only medical record on the flock is routine deworm with Levamisole hydrochloride.

Physical examination of the lamb revealed that the lamb was healthy, although slightly underweight. The hind limbs were disproportionate (Plate 1) with the left hind limb bowing outward and the right hind limb limb appeared normal from the pelvis to the distal tibia. However, the tarsus, metatarsus and phalanges were deformed. The right hoof was absent only leaving behind a cutaneous structure, and a wound on its abaxial surface. Other parameters were within normal limit of the age of the lamb.

About $3 \mathrm{mls}$ of blood was collected through jugular venipuncture from the lamb and a flock mate of the same age but with no deformity (Control) for the determination of the serum values of alkaline phosphatase (ALP) alanine transaminase (ALT) and aspartate transaminase (AST). Also both lateral and antero-posterior radiographs of the hind limb were obtained using a Philippractex 20mA portable machine radiographs of the hind limb were obtained using a Philip practex $20 \mathrm{~mA}$ portable machine.

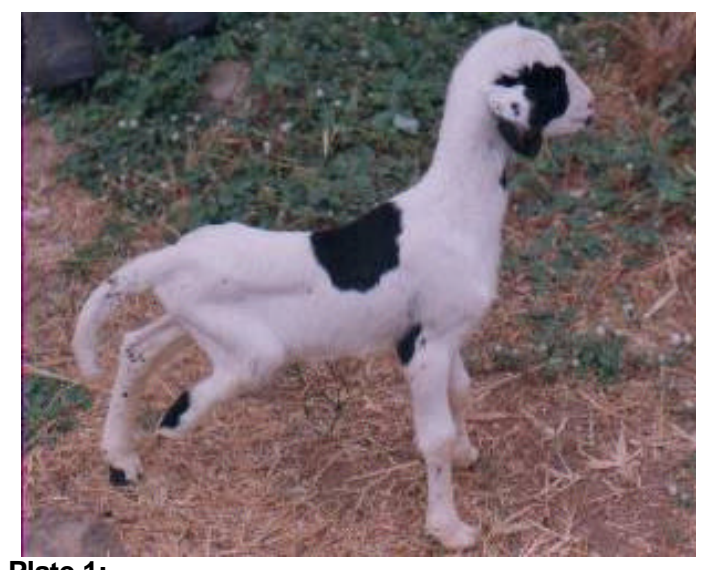

Plate 1:

A three week old WAD Lamb with disproportionate hind limb

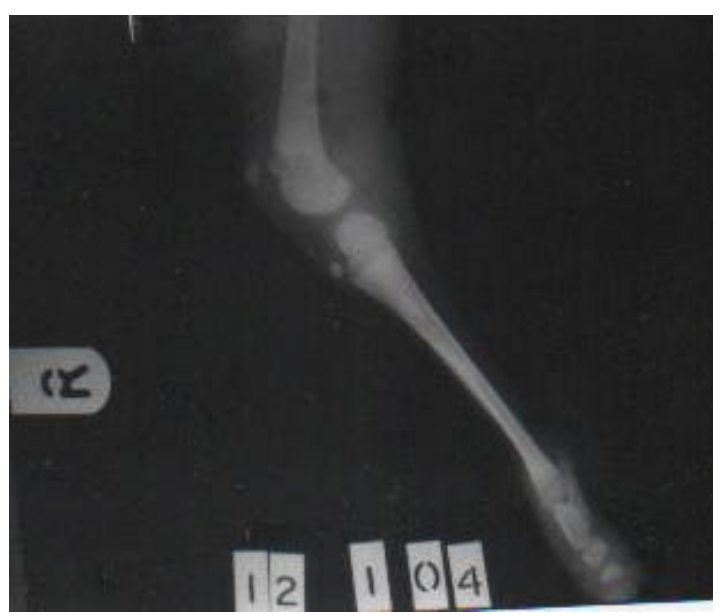

Plate 2:

Lateral Radiograph of the right, hind limb showing deformed tarsal bones, and metatarsal bones.

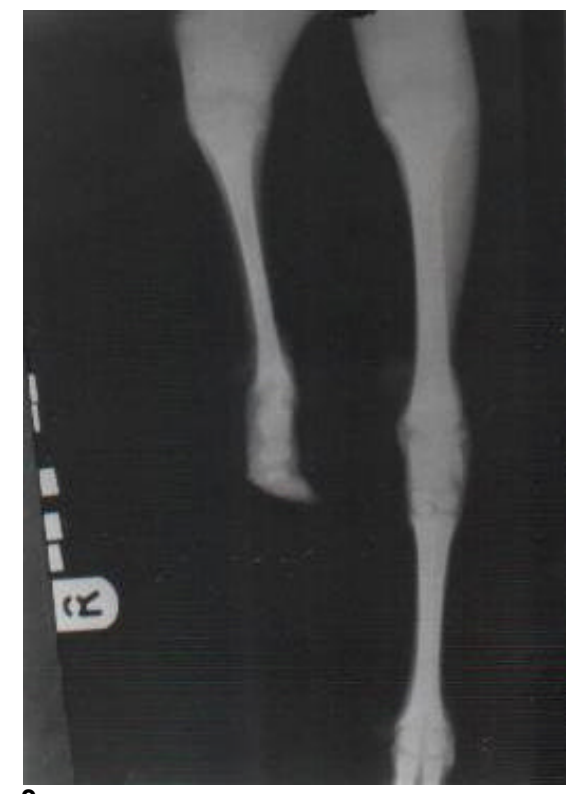

Plate 3:

Anteroposterior Radiograph comparing the left hind limb with the right hind limb

\section{Results of Laboratory Investigations}

The serum ALP, AST and ALT were respectively higher in the achondroplastic lamb (15 K.A.u/L; 47 i.u/L; 43 i.u/L) compared with the control lamb (10 K.A.U/L; 36 i.uK; 34 i.u/L). Radiological examination (Plates 2 \& 3) showed that the physes are still opened. The left tibiofibula bone appeared slightly expanded with thinner cortex. The right tarsal bones were very small and laterally compressed while the lateral sesamoid bone was absent. The right metatarsal bones were rudimentary and were only represented by two small fused bones ofabou3mm in diameter. The phalangeal bones were absent and were only replaced by soft' tissue with concave bowing of the hoof structure. In addition, there was moderate swelling of the entire tarsus.

\section{DISCUSSION AND CONCLUSION}

Chondrodysplasia has been reported in other breeds of sheep either as a single entity or in association with other defects (Wray et al 1971; Rook et al, 1988). However, this appears to be the first reported case of achondroplastic syndrome in West African Dwarf Lamb. Although the exact cause of the defect is unknown, it is unlikely that the syndrome described was caused by nutritional factors, drugs or disease. Some transmissible diseases have been shown to cause deformed foetus such as vibriofoeui listeria monocyto genes and parainfluenza virus (Hore et al, 1973).

Congenital defect may be caused by intrauterine environment or may be hereditary. (Rook et a!, 1988). The most likely explanation is that this condition is of genetic origin and could either be due to a recessive gene or a dominant gene with incomplete penetration. Routine hematology studies and serum 
biochemical analyses are often done as part of investigation of hereditary chondrodysplasia. Rook et al, (1988) reported a slight increase in serum ALP between chondroplastic lamb and normal lambs. In this report, the serum ALP, AST and ALT were respectively higher in the achondroplastic lamb compared to the control lamb.

It is probable that this defect affects the development of the bone or the cartilage. This is so because the tarsal and metatarsal bones were rudimentary while the phalanges were replaced by soft tissue structure.

\section{REFERENCES}

Dennis S.M (1974). A survey of congenital defect of sheep. Vet Res. 95:4,88-490.

Dennis S.M (1975). Early mortality in Westgrn Auitralla Congonitl Dsfcct Aust. Vet. 3.50:80-82.

Hore, D.E; Smith, 'O; Snowdon, W; Uren 3. (1973). An investigation of viral and bacterial agents associated with ovine perinated mortality Aus. Vet. 3.49:190-195.

Leipold, H.W; Dennis, S.M. Huston K. (1972). Congenital Defects of Cattle. Nature, cause and effect. Adv. Vet Sec. Comp. Med. 16:142-151

Nigel palmer (1993). Diseases of Bones In pathology of domestic Animals (Jubb, KV.F; Peter, Kennedy, Nigel Palmor eds) 4th edition Academic Press. Santiago Pp.27-31.

Rook, J.S, Kopcha, M; Spaulding K et al (1986). The spider syndrome A report on one pure- bred flock. Comp. Cont. Edur. Prac. Vet. 8:402-408.

Rooks, J.S; Allan, L.T; Janver, K; Yamini, B. (1988) Diagnosis of hereditary chondrodysplasia (Spider Lamb syndrome in sheep. J.A.V.M.A 193: 713-718

Silence, O.O; Horton, W.A; Rimoin, D.L. (1979) Morphologic studies in the skeletal dysplasias. A review. Am. 3. Pathol. 96:812-859.

Saperstein, G; Leipold, H.W; Dennis, S.M (1975). Congenital defects of sheep. J.AN.M.A 167:314-322. Wray, C; Mathieson, A.O; Cop land, AN (1971). An achondroplastic syndrome in South Country Cheviot Sheep. Vet. Rec. .88:521-522.

*Author for correspondence (E-mail: ade_vsr@hotmail.com) 